\title{
Modeling an OBS Node under Critical Load and High Utilization Conditions
}

\author{
Jianan Zhang, Eric W. M. Wong, Senior Member, IEEE, and Moshe Zukerman, Fellow, IEEE
}

\begin{abstract}
We consider a bufferless optical burst switching optical cross connect modeled as a continuous-time Markov chain based on a generalized Engset model. We focus specifically on critical load and other given levels of high utilization conditions and evaluate the required number of wavelength channels per cable to keep the blocking probability below a given level. We observe that although a large number of wavelength channels per cable are required to achieve low blocking probability under critical load condition, the required number of such channels decreases significantly if the utilization is allowed to drop to $90 \%$ or $80 \%$. We also propose a new blocking probability approximation that is more accurate than previous approximations under critical load condition.
\end{abstract}

Index Terms-Blocking probability, optical burst switching (OBS), generalized Engset formula, critical load, high utilization.

\section{INTRODUCTION}

$\mathbf{O}$ PTICAL fibers with wavelength division multiplexing (WDM) technology provide large bandwidth for today's Internet. Optical burst switching (OBS) [1] is one potential switching technology for WDM networks. In OBS, traffic is carried by bursts, each of which consists of packets that have the same destination.

It has been argued that since OBS allows for burst collisions, link utilization may have to be sacrificed to meet the required quality of service (QoS) [2]. If the number of wavelength channels per cable is small, blocking probability is usually high under critical load condition where offered load equals to transmission capacity. However, it can be reduced as the number of wavelength channels increases [3]. The generalized Engset model can be applied to evaluate the blocking probability at an OXC in bufferless OBS systems [4-10].

We aim to investigate the number of wavelength channels per cable required to keep the blocking probability at an $\mathrm{OXC}$ below a given level under critical load and other given levels of high utilization conditions. However, it is computationally prohibitive to obtain the exact solutions for cases where the number of channels is too large. Therefore, we propose a new blocking probability approximation. This approximation is more accurate than the others under critical load condition.

We demonstrate that OBS can achieve high utilization under a reasonable number of wavelength channels per cable. However, a large number of channels are required to achieve low blocking probability under critical load condition.

Manuscript received December 15, 2011. The associate editor coordinating the review of this letter and approving it for publication was X. Cao.

J. Zhang is with the Electronic Engineering Department, Tsinghua Univ. Beijing, PRC. E. W. M. Wong and M. Zukerman are with the Electronic Engineering Department, City Univ. of Hong Kong, Hong Kong SAR, PRC.

\section{OXC MODELING}

Assume for simplicity that each cable has $F$ optical fibers, each of which carries $W$ wavelengths. Consider an output cable of an OXC with total $F W$ wavelength channels, out of which $K$ wavelength channels, referred to as servers, are available to transmit an arriving burst on a given wavelength, where $K=\alpha F W$. With full wavelength conversion, $\alpha=1$; with no wavelength conversion, $\alpha=1 / W$; with partial wavelength conversion, $1 / W<\alpha<1$. Collision occurs when a burst on a given wavelength requires one of the servers, but all the servers are busy transmitting other bursts, in which case the burst is dumped. Relevant input wavelength channels that may carry bursts directed to the servers are referred to as $M$ sources. The value of $K$ (or equivalently, since $\alpha$ is known, the total number of wavelength channels in a cable $-F W$ ) is key to efficiency, and therefore the focus of this paper.

Each input wavelength channel of the OXC transmits bursts as an on/off process. On-time refers to burst transmission time and off-time refers to idle time between bursts. Mean onand off-times are $1 / \mu$ and $1 / \lambda$, respectively. For tractability, we assume that both on- and off-times follow exponential distribution, which does not introduce significant errors [11]. Based on the generalized Engset model, the system can be modeled as a two-dimensional Markov chain [4-7]. The first dimension stands for the number of busy sources (bursts being transmitted, and occupying both input and output wavelength channels). The second dimension stands for the number of frozen sources (bursts being dumped, and occupying the input wavelength channels). Free sources refer to input channels during their periods of the off-time. Although computation time can be reduced [7], for large values of $M$ and $K$, an exact solution is intractable because of the large state space.

\section{A NEW APPROXIMATION}

Consider a free or frozen source in the generalized Engset model, with probability $p_{b}$, it is frozen and the expected time duration until the next burst arrival is $1 / \mu+1 / \lambda$. With probability $1-p_{b}$, it is free and the expected time duration until the next burst arrival is $1 / \lambda$. Mean time duration until the next burst arrival for a free or frozen source equals to:

$$
\frac{1}{\lambda^{*}}=\frac{1}{\lambda}+\frac{p_{b}}{\mu} .
$$

By lengthening the off-time of the Engset model, where free sources refer to free or frozen sources in the generalized Engset model, we obtain a blocking probability approximation. In [8], the off-time is lengthened to (1); the offered load of 
this modified Engset model equals to that of the generalized Engset model. The blocking probability is given by the Engset formula, which is computationally stable and efficient:

$$
p_{b}=\operatorname{Eng}\left(M, K, \lambda^{*}, \mu\right)=\frac{\left(\begin{array}{c}
M-1 \\
K
\end{array}\right)\left(\frac{\lambda^{*}}{\mu}\right)^{K}}{\sum_{k=0}^{K}\left(\begin{array}{c}
M-1 \\
k
\end{array}\right)\left(\frac{\lambda^{*}}{\mu}\right)^{k}} .
$$

Although [8] accurately considers the offered load, a lack of accurate matching of other traffic statistics renders it not very accurate especially under critical loading. Next, we introduce the following lengthened off-time.

$$
\frac{1}{\lambda^{*}}=\frac{1}{\lambda}+\frac{p_{b}}{\left(1-p_{b}\right) \mu} .
$$

It considers the sum of frozen times of a geometric sequence of failed transmissions. In critical load and lighter load conditions, it is generally more accurate to evaluate the time interval between two consecutive successful transmissions compared with (1), which assumes that at most one frozen period and one idle period may exist between two consecutive successful transmissions. Note that [9], which adds the off-times considering consecutive failures, leads to far worse approximations, because [9] leads to much lower offered load evaluations. The new approximation (3) provides a balance between the evaluations of offered load and time duration between two consecutive successful transmissions. In underload conditions $(U=M \lambda /[K(\lambda+\mu)]<1)$, the new approximation (3) slightly overestimates the blocking probability. We use the multiplier $2 /\left(1+U^{2}\right)$ to further lengthen the off-time to achieve higher accuracy. Moreover, it is more accurate than the state dependent approximation [10] in critical load condition $(U=1)$, because the servers are occupied for nearly all the time, leading to a situation that only a small number of neighboring states (including the congestion states) have high probability and that the benefit of the state dependent approximation is small.

In overload conditions, the carried loads estimated by these approximations and the carried load in the exact system are all close to $K$. In such cases, (3) and [9] underestimate the offered load and thus underestimate the blocking probability; [8] and [10] match the offered load and thus are more accurate. To narrow the gap between the offered loads in the exact system and the approximation (3), the multiplier $\left[\left(1-p_{b}\right) U^{a}+p_{b}\right] / U^{a}$ is introduced to shorten the off-time. We find that $a=5$ gives the most accurate results through empirical tests. As $U$ increases, the modified off-time approaches (1), which improves the evaluations of the offered load.

$$
\frac{1}{\lambda^{*}}=\left\{\begin{array}{ll}
\frac{1}{\lambda}+\frac{p_{b}}{\left(1-p_{b}\right) \mu} \frac{2}{1+U^{2}} & U \leq 1 \\
\frac{1}{\lambda}+\frac{\left.p_{b}\right)}{\left(1-p_{b}\right) \mu} \frac{\left(1-p_{b}\right) U^{5}+p_{b}}{U^{5}} & U>1
\end{array}, U=\frac{M \lambda}{K(\lambda+\mu)} .\right.
$$

Extensive tests have shown that the approximation (4) is generally more accurate than the approximations of [8-10] under critical load condition and under somewhat lower traffic loads as long as the utilization is above $70 \%$ and $K \geq 50$.

The blocking probability can be obtained by solving equations (2) and (4). Next, we demonstrate that there exists one unique solution. Substituting $1 / \lambda^{*}$ by $x$ in (4), and deducting $x$ from both sides of (4), we obtain:
TABLE I

COMPARISON OF RELATIVE ERRORS OF APPROXIMATIONS UNDER CRITICAL LOADING, $1.2 \leq M / K \leq 10,50 \leq K \leq 150$.

\begin{tabular}{ccccc}
\hline Relative error & {$[\mathbf{8}]$} & {$[\mathbf{9}]$} & {$[\mathbf{1 0}]$} & New \\
\hline Average & 0.014401 & -0.290330 & 0.011004 & 0.003383 \\
\hline Standard deviation & 0.009964 & 0.048215 & 0.007405 & 0.003885 \\
\hline
\end{tabular}

TABLE II

\begin{tabular}{|c|c|c|c|c|c|}
\hline$(\lambda, M, K)$ & Exact & $\begin{array}{c}{[8]} \\
\text { (\% error) }\end{array}$ & $\begin{array}{c}{[9]} \\
\text { (\% error) }\end{array}$ & $\begin{array}{c}{[10]} \\
\text { (\% error) }\end{array}$ & $\begin{array}{c}\text { New } \\
\text { (\% error) }\end{array}$ \\
\hline$(1,100,50)$ & 0.0903 & $\begin{array}{l}0.0932 \\
(3.2 \%)\end{array}$ & $\begin{array}{c}0.0707 \\
(-21.7 \%)\end{array}$ & $\begin{array}{l}0.0922 \\
(2.1 \%)\end{array}$ & $\begin{array}{l}0.0905 \\
(0.2 \%)\end{array}$ \\
\hline$(1,200,100)$ & 0.0656 & & $\begin{array}{c}0.0512 \\
(-22.0 \%)\end{array}$ & $\begin{array}{l}0.0670 \\
(2.1 \%)\end{array}$ & $\begin{array}{l}0.0661 \\
(0.8 \%)\end{array}$ \\
\hline$(1,300,150)$ & 0.054 & $\begin{array}{l}0.0557 \\
(2.8 \%)\end{array}$ & $\begin{array}{c}0.0422 \\
(-22.1 \%)\end{array}$ & $\begin{array}{l}0.0554 \\
(2.2 \%)\end{array}$ & $\begin{array}{l}0.0547 \\
(0.9 \%)\end{array}$ \\
\hline$(0.1,550,50)$ & 0.1027 & $\begin{array}{l}0.1032 \\
(0.5 \%)\end{array}$ & $\begin{array}{c}0.0693 \\
(-32.5 \%)\end{array}$ & $\begin{array}{l}0.1031 \\
(0.4 \%)\end{array}$ & $\begin{array}{l}0.1027 \\
(0.0 \%)\end{array}$ \\
\hline$(0.1,1100,100)$ & 0.0742 & $\begin{array}{l}0.0746 \\
(0.5 \%)\end{array}$ & $\begin{array}{c}0.0497 \\
(-33.0 \%)\end{array}$ & $\begin{array}{l}0.0745 \\
(0.4 \%)\end{array}$ & $\begin{array}{l}0.0743 \\
(0.1 \%)\end{array}$ \\
\hline$(0.1$, & 0.06 & $\begin{array}{l}0.0615 \\
(0.5 \%)\end{array}$ & $\begin{array}{c}0.0408 \\
(-33.3 \%)\end{array}$ & $\begin{array}{l}0.0615 \\
(0.5 \%)\end{array}$ & $\begin{array}{l}0.0613 \\
(0.2 \%)\end{array}$ \\
\hline
\end{tabular}

COMPARISON OF APPROXIMATIONS WITH RELATIVE ERRORS IN BRACKETS UNDER CRITICAL LOADING, $\mu=1$.

$$
f(x)=\left\{\begin{array}{ll}
\frac{1}{\lambda}+\frac{p_{b}}{\left(1-p_{b}\right) \mu} \frac{2}{1+U^{2}}-x & U \leq 1 \\
\frac{1}{\lambda}+\frac{p_{b}}{\mu}\left[1+\frac{p_{b}}{\left(1-p_{b}\right) U^{5}}\right]-x & U>1
\end{array} .\right.
$$

Regard $p_{b}$ as a function of $x$. As $x$ increases, $p_{b}(x)$ and $1 /[1-$ $p_{b}(x)$ ] decreases. Therefore, $f(x)$ is a monotonic decreasing function of $x$. Besides, $f(x)>0$ when $x$ approaches $0 ; f(x)<$ 0 when $x$ increases and $p_{b}(x)$ approaches 0 . There exists a unique solution for $f(x)=0$. We apply binary search algorithm to obtain $x$, and then use (2) to obtain the blocking probability.

\section{NumericAl Results}

In this section we numerically verify the accuracy of the new approximation and then evaluate the number of wavelength channels per cable to maintain the blocking probability below a given level at an OBS OXC.

We have considered 2200 different cases under critical load condition, where $50 \leq K \leq 150$ and $1.2 \leq M / K \leq 10$. In all these cases, the new approximation achieves the most accurate results. In Table I, we present the average and standard deviation of the relative error of each approximation, and observe that, on average, the new approximation reduces the relative error by around $70 \%$, and has lower standard deviation of the relative error compared with the next most accurate approximation. From these 2200 results, selected representative cases are presented in Table II.

The approximation is fairly accurate under critical load condition except when $1<M / K<1.2$. As $M$ approaches $K, \lambda$ rapidly increases (reaches infinity for $M=K$ ). In such a case, there is an increasingly significant difference between blocking probabilities of the generalized Engset model and the Engset model with the same $M, K, \lambda$ and $\mu$. Our simple rule based on (4) of increasing the off-time can not capture this effect well. Nevertheless, in practice, an OXC has multiple input and output ports, and each input port may provide bursts to an 
output port, so it is likely that $M$ is at least several times larger than $K$, in which case, our new approximation is accurate.

The blocking probability approximation is upper bounded by the Engset formula, because we lengthen the off-time in the Engset formula to obtain the approximation. It is supported by the fact that the exact blocking probability is also upper bounded by the Engset formula. Intuitively, in the generalized Engset system, the only difference from the Engset system is that there is an extra delay period after a blocking event within which the blocked source is frozen. This delay reduces burst arrival rate and hence blocking probability.

Next we discuss how many sources and servers are required in order to keep the blocking probability below $10^{-6}$ under critical load and other given levels of high utilization conditions. We compare different cases where $M / K=$ 2, 10, 30, 100, depicted in Table III. Numbers followed by (a) are obtained by approximations and the others are obtained by exact solutions. We observe that as the utilization decreases, the required number of servers decreases significantly.

TABLE III

MINIMUM $K$ TO KEEP BLOCKING PROBABILITY BELOW $10^{-6}$.

\begin{tabular}{|c|c|c|c|c|c|}
\hline Utilization & $100 \%$ & $90 \%$ & $80 \%$ & $70 \%$ & $60 \%$ \\
\hline$M=2 K$ & $5.14 * 10^{11}(a)$ & 967 & 253 & 113 & 63 \\
\hline$M=10 K$ & $6.20 * 10^{11}(a)$ & $1567(a)$ & 393 & 169 & 90 \\
\hline$M=30 K$ & $6.31 * 10^{11}(a)$ & $1664(a)$ & 416 & 178 & 94 \\
\hline$M=100 K$ & $6.35 * 10^{11}(a)$ & $1697(a)$ & $424(a)$ & 181 & 96 \\
\hline
\end{tabular}

Figure 1 and Table IV demonstrate that the minimum $K$ $\left(K_{\text {min }}\right)$ is monotonically increasing in $M / K$ in order to keep the blocking probability below $10^{-6}$. We use approximations for $M / K=100$ cases in the figure, and use exact solutions for the other cases in the figure and Table IV. For a given utilization, $K_{\min }$ keeps stable when $M / K>40$, and approaches $K_{\min }$ obtained in Erlang loss system, which corresponds to the $M$ approaching infinity case. Moreover, we observe that if $M / K$ is doubled, the increment in $K_{\min }$ is approximately half of the decrement in $K_{\min }$ if $M / K$ reduces to half.

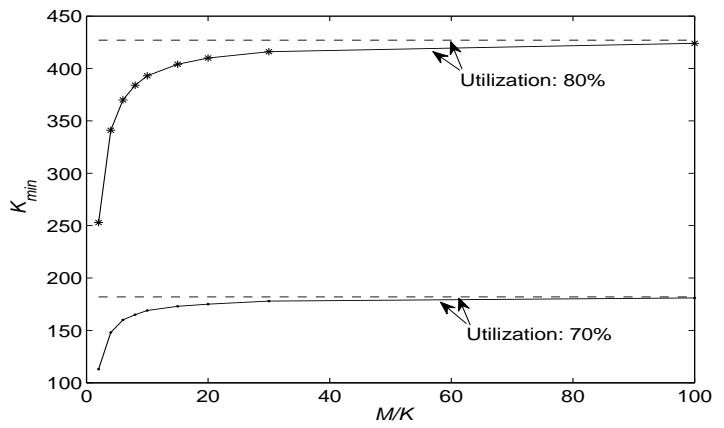

Fig. 1. Solid lines are $K_{\min }$ in the generalized Engset system to keep the blocking probability below $10^{-6}$ for different $M / K$. Dash lines are $K_{\min }$ in the Erlang loss system with the same blocking probability constraint.

For a given OXC and wavelength converter, $\alpha$ is known. We can obtain the number of wavelength channels per cable $(F W)$ based on $K$. Currently, a cable may contain over a hundred optical fibers, and each WDM fiber may carry over a hundred wavelengths. The numbers keep increasing with
TABLE IV

MiNimum $K$ TO KEEP THE BLOCKING PROBABILITY BELOW $10^{-6}$ VS. $M / K$ WHEN UTILIZATION IS $80 \%$ OR $70 \%$.

\begin{tabular}{ccc}
\hline Utilization & $80 \%$ & $70 \%$ \\
\hline$K_{\min }(M / K=2)$ & 253 & 113 \\
\hline$K_{\min }(M / K=4)$ & 341 & 148 \\
\hline$K_{\min }(M / K=8)$ & 384 & 165 \\
\hline$K_{\min }(M / K=16)$ & 406 & 174 \\
\hline
\end{tabular}

the development of manufacturing techniques of optical fibers. Therefore, higher utilization is achievable in the future under the same blocking probability constraint.

\section{CONCLuSion}

We have studied the required number of wavelength channels per cable to maintain the blocking probability at an OBS OXC below a given level under critical load and other given levels of high utilization conditions. For cases where the number of required output wavelength channels is large, we have derived and used a new blocking probability approximation that is fairly accurate under critical load condition. To maintain the blocking probability below $10^{-6}$, a large number of channels per cable are required under critical load condition. However, the required number decreases significantly in underload conditions where the utilization is still high. Our choice of $10^{-6}$ blocking probability for any given OXC is sufficiently small to maintain acceptable end-to-end QoS, and we have demonstrated that OBS can be reasonably efficient under this condition. Although $100 \%$ utilization for OBS is not likely achievable in the foreseeable future, reasonable utilization levels in the range of $70 \%-80 \%$ are achievable.

\section{REFERENCES}

[1] Y. Chen, C. Qiao, and X. Yu, "Optical burst switching: a new area in optical networking research," IEEE Network, vol. 18, no. 3, pp. $16-$ 23, May-Jun. 2004

[2] V. S. Puttasubbappa and H. G. Perros, "Performance analysis of limitedrange wavelength conversion in an OBS switch," J. Telecommun. systems, vol. 41, no. 2-3, pp. 2961-2973, Oct. 2006.

[3] E. W. M. Wong, L. L. H. Andrew, T. Cui, B. Moran, A. Zalesky, R. S. Tucker, and M. Zukerman, "Towards a bufferless optical Internet," $J$. Lightwave Technol., vol. 27, no. 14, pp. 2817-2833, Jul. 2009.

[4] M. Zukerman, E. W. M. Wong, Z. Rosberg, G. M. Lee, and H. L. Vu, "On teletraffic applications to OBS," IEEE Commun. Lett., vol. 8, no. 2, pp. 116-118, Feb. 2004.

[5] A. Detti, V. Eramo, and M. Listanti, "Performance evaluation of a new technique for IP support in a WDM optical network: optical composite burst switching (OCBS)," J. Lightwave Technol., vol. 20, no. 2, pp. 154 -165 , Feb. 2002.

[6] H. Øverby, "Performance modelling of optical packet switched networks with the Engset traffic model," Optics Express, vol. 13, pp. 1685 - 1695, Mar. 2005.

[7] N. Akar and Y. Gunalay, "Stochastic analysis of finite population bufferless multiplexing in optical packet/burst switching systems," IEICE Trans. Commun., pp. 342-345, Feb. 2007.

[8] H. L. Vu, A. Zalesky, E. W. M. Wong, Z. Rosberg, S. M. H. Bilgrami, M. Zukerman, and R. S. Tucker, "Scalable performance evaluation of a hybrid optical switch," J. Lightwave Technol., vol. 23, no. 10, pp. 2961-2973, Oct. 2005.

[9] E. W. M. Wong, A. Zalesky, and M. Zukerman, "On generalizations of the Engset model," IEEE Commun. Lett., vol. 11, no. 4, pp. 360-362, Apr. 2007.

[10] - "A state-dependent approximation for the generalized Engset model," IEEE Commun. Lett., vol. 13, no. 12, pp. 962-964, Dec. 2009.

[11] J. Zhang, Y. Peng, E. W. M. Wong, and M. Zukerman, "Sensitivity of blocking probability in the generalized Engset model for OBS," IEEE Commun. Lett., vol. 15, no. 11, pp. 1243-1245, Nov. 2011. 